\title{
Developing effective and efficient genomic educational tools for our diverse population
}

\author{
Julia Wynn ${ }^{1}$, Wendy K. Chung ${ }^{1,2}$ \\ ${ }^{1}$ Department of Pediatrics, ${ }^{2}$ Department of Medicine, Columbia University Irving Medical Center, New York, NY, USA \\ Correspondence to: Wendy K. Chung, MD, PhD. 1150 St. Nicholas Ave., Russ Berrie Pavilion 6th Fl., Room 620, New York, NY 10032, USA. \\ Email: wkc15@columbia.edu. \\ Provenance: This is an invited article commissioned by the Section Editor Dr. Xiao Li, MD (Department of Urology, Jiangsu Cancer Hospital \& \\ Jiangsu Institute of Cancer Research \& Nanjing Medical University Affiliated Cancer Hospital, Nanjing, China). \\ Comment on: Hurtado-de-Mendoza A, Graves KD, Gómez-Trillos S, et al. Developing a culturally targeted video to enhance the use of genetic \\ counseling in Latina women at increased risk for hereditary breast and ovarian cancer. J Community Genet 2019. [Epub ahead of print].
}

Submitted Oct 09, 2019. Accepted for publication Oct 22, 2019.

doi: $10.21037 /$ atm.2019.10.98

View this article at: http://dx.doi.org/10.21037/atm.2019.10.98

As genetics is increasingly integrated into medicine, there is a pressing need for scalable genomic education that address the needs of our diverse population. Traditionally, genomic education and counseling are provided by genetic counselors in-person, one-on-one, in a pre- and posttesting model. While this is the gold standard, it is not scalable nor is it necessarily what all patients need or want. Furthermore, traditional genetic counseling may not be the most effective model for the patients, particularly when there are language and/or cultural differences between the provider and patient (1). Alternative educational tools and delivery models have the potential to meet the needs of the patient and the provider while not placing excessive burden on the healthcare system. These tools should address the different cultures, languages, health and genetic literacy, access and technological capabilities of the population. Failure to address these different aspects will result in exacerbating rather than ameliorating healthcare disparities.

Hurtado-de-Mendoza et al. recently created a genetic education tool, "Developing a culturally targeted video to enhance the use of genetic counseling in Latina women at increased risk for hereditary breast and ovarian cancer," published in the May 18th edition of the Journal of Patient Education and Counseling. The authors eloquently describe their process of developing a culturally targeted video that delivered education in Spanish for hereditary breast and ovarian cancer (HBOC) genetic testing for a low literacy Latina population. The goal of this tool is to enhance the use of genetic cancer risk assessment (GCRA) services by improving understanding of HBOC risk and how to manage it. The authors' experiences provide a guide for the development of future tools.

Hurtado-de-Mendoza et al. (2) began by identifying a narrative format as an accessible and effective educational method for their target population based on review of current literature. The authors then conducted interviews with stakeholders, healthcare providers and Latinas at risk for HBOC, to identify critical facts, common information gaps, motivators and barriers to pursue GCRA, and the language the target community uses to describe HBOC and GCRA. The video content and scripts were informed by these interviews. For example, the authors decided to use "gene alteration" rather than "mutation" which has negative connotations within this community or "gene change" which the authors were concerned may mislead viewers about the permanence of a pathogenic variant. They also identified potential storylines to illustrate barriers to GRCA including the stigma of cancer in the Latino community and financial constraints that limit access to care. The authors were careful to point out that these words and issues are specific to this community and may not translate to other populations. In an effort to balance everyday language with scientific precision, patients depicted in the video used plain and sometime slightly inaccurate language while the healthcare providers maintained scientifically accurate language. After development of the script, the authors 
solicited stakeholder opinions through a focus group and made iterative improvements including emphasizing pathways to receive care for those under or not insured. The final script delivered the key messages about HBOC and GRCA for this community rather an exhaustive list of all risks and benefits of GRCA.

The final 18 -minute video was produced by a professional video production company with experience developing documentary films. The video is in Spanish and has Latino actors to help the viewers relate. Community health workers and patient navigators who work with the Latino community viewed the video as part of their participation in a genetics workshop and completed a survey assessing the video. They were highly satisfied with the video and the effectiveness of the message, and most indicated a plan to share it with their patients. A study is underway to assess if viewing the video increases the uptake of GCRA in at risk Latina women.

A key part of Hurtado-de-Mendoza et al. development process was the engagement of all stakeholders-in particular the target audience-early on and throughout the development. This maximizes the potential for success of the tool by helping to ensure the tool delivers the information needed and valued by the target community in a relatable and accessible manner. While involving the community can be costly, time consuming and result in a need for more revisions, it is critical. Genetic counselors and healthcare providers have specific training in communicating complex information. However, we do this face-to-face, where we can assess facial expressions and ask questions to assess understanding; strategies that are not possible with educational videos. User experience testing during the development serves as this check by showing how a user navigates and engages with the tool and interprets the content. Developers can use this feedback to iteratively improve a tool resulting in a more effective final product.

This paper demonstrates how collaborations can produce a tool that has the appropriate balance between information and engaging content. Hurtado-de-Mendoza et al. developed the videos in collaboration with professional video developers and actors. Professional video and web developers understand how to build content to capture and maintain a viewer's attention to deliver the message. Healthcare providers are not formally trained to develop videos and webpages and benefit from collaboration with educators. These collaborations may not always be easy, and goals and methods may differ. Hurtado-de-Mendoza et al. reported challenges in working with fast-paced production timelines and balancing artistic liberties with accuracy of the information.

While not completed by these authors, it may also be beneficial to collaborate with experts in the use of digital technology for education. Their expertise in designing digital learning tools could help identify strategies to maximize learning with different multimedia learning environments. These collaborations can help us to produce content beyond videos such as chat bots that use artificial intelligence to deliver interactive content.

To save money and resources, there is often a desire to develop a single tool for a given topic. While tempting, our experience has taught us that rarely is there one solution that works for everyone, and messages are most effective when they are tailored for specific patient groups. Hurtado-de-Mendoza et al. identified a specific population and a specific topic and tailored the message for this audience. While their tool is purposely limited in its use, their systematic process should be used as a guide for developing other resources for other topics and target audiences.

This paper details the labor-intensive process of developing genomic video education. Given the cost and time involved in developing educational modules and delivery platforms and the need to develop different tools specific to the needs of the target audience, we must share these tools freely. Similar to data repositories for genomic data, we also need a repository of educational tools annotated with the clinical use case, target audience and whether or not the tools have evidence of effectiveness. Technology has the potential to help us meet the diverse needs of our patients and to deliver scalable genomic education. We should embrace this potential by developing engaging and effective tools.

\section{Acknowledgments}

None.

\section{Footnotes}

Conflicts of Interest: The authors have no conflicts of interest to declare.

Etbical Statement: The authors are accountable for all aspects of the work in ensuring that questions related to the accuracy or integrity of any part of the work are 
appropriately investigated and resolved.

\section{References}

1. Joseph G, Pasick RJ, Schillinger D, et al. Information Mismatch: Cancer Risk Counseling with Diverse

Cite this article as: Wynn J, Chung WK. Developing effective and efficient genomic educational tools for our diverse population. Ann Transl Med 2019;7(Suppl 8):S304. doi: 10.21037/atm.2019.10.98
Underserved Patients. J Genet Couns 2017;26:1090-104.

2. Hurtado-de-Mendoza A, Graves KD, Gómez-Trillos S, et al. Developing a culturally targeted video to enhance the use of genetic counseling in Latina women at increased risk for hereditary breast and ovarian cancer. J Community Genet 2019. [Epub ahead of print]. 